\section{Patologías orales en la mujer gestante. Revisión de la literatura}

\section{Oral diseases in pregnant women. Literature review}

\begin{abstract}
Resumen
El embarazo constituye un período de la vida de la mujer donde suceden cambios tanto de su cuerpo como en su entorno. Los cambios hormonales que sufre la mujer durante el estado de gravidez pueden llegar a afectar su salud bucal desencadenándose una serie de alteraciones a nivel gingival lo cual propicia la presencia de enfermedades bucodentales. Se busca mediante esta revisión bibliográfica actualizar conocimientos acerca de las diferentes patologías bucales en la mujer gestante. Se revisaron las bases de datos Medline, SciELO y Google Scholar en busca de estudios de casos, ensayos clínicos, metanálisis, revisiones sistemáticas y revisiones de literatura publicadas en los últimos 8 ańos, sobre las diferentes patologías bucales que se presentan en las mujeres embarazadas, empleando como palabras clave mujeres embarazadas, enfermedades bucodentales, salud bucal y sus homólogos en inglés. Se obtuvieron 72 artículos, después de la lectura y exclusión, la selección arrojó 42 artículos considerados adecuados para cumplir con los objetivos planteados. La adopción de buenos hábitos de higiene oral y consultas periódicas al odontólogo pueden reducir los efectos adversos durante el embarazo; aunque otros investigadores afirman la ausencia de cambios durante la gestación en relación con la salud periodontal. El acompañamiento y orientación durante la gestación requiere involucrar cuidados bucales desde inicios de esta etapa hasta el nacimiento del bebe.
\end{abstract}

Palabras clave: Enfermedades bucales; Mujeres embarazadas; Salud bucal.

\section{Artículo de Revisión}

Guiselle González Chavarría 1,a, Andrea Revelo Domínguez ${ }^{1, a}$, Isabel Chiriboga Sánchez ${ }^{1, a}$, Jonathan Eduardo Aldas Ramírez 1,a, Ana del Carmen Armas Vega ${ }^{1, b}$

1 Universidad Tecnológica Equinoccial. Facultad de Odontología. Quito, Ecuador.

a Odontólogo.

${ }^{b} \mathrm{PhD}$ en Operatoria Dental.

Correspondencia:

Guiselle González Chavarría

Correo electrónico: gisel_gico@hotmail.com Sangolquí, Urbanización Nacional del Banco del Fomento, Manzana 26 lote 2. Quito, Ecuador.

\section{Coautores: \\ Andrea Revelo Domínguez Andrearevelo94@hotmail.com Isabel Chiriboga Sánchez isach.odonto@gmail.com Jonathan Eduardo Aldas Ramírez J.Aldas_dental@outlook.com Ana del Carmen Armas Vega ana_del_ec@yahoo.es}

Conflicto de intereses: Los autores declaran no tener conflictos de interés.

Fuente de financiamiento: Universidad Tecnológica Equinoccial

Fecha de recepción: 06/03/18

Fecha de aceptación: 16/07/18 
their relevant information. Most authors conclude that good oral hygiene habits and regular visits to the dentist can reduce adverse effects during pregnancy, although other researchers do not affirm the existence of a relationship between periodontal disease and adverse effects, but emphasize that periodontal treatment can be done during pregnancy to relieve the symptoms. Due to the changes suffered by women during the management, they require special and exclusive treatment, in addition to guiding her about oral care for both her and him after childbirth.

Keywords: Mouth diseases; Oral health; Pregnant women.

\section{Introducción}

La salud oral durante el período de gestación es de interés para madres y profesionales de la salud ${ }^{1,2}$. El estado de gravidez viene acompañado de una serie de cambios fisiológicos, patológicos y psicológicos convirtiendo a la mujer gestante en paciente con demanda de cuidados especiales, necesarios desde inicios del embarazo ${ }^{3,4}$ para evitar la presencia de patologías orales ${ }^{5-7}$.

Si bien el embarazo no es una enfermedad, durante su transcurso existen cambios hormonales ${ }^{4}$, que desencadenan la presencia de alteraciones bucales ${ }^{3,8}$. La alta prevalencia de caries es considerada la principal causa de pérdida dental convirtiéndola en un problema de salud pública ${ }^{9}$, resultado de la ruptura del ecosistema microbiano, asociado a una baja capacidad de limpieza y lubricación salival ${ }^{4}$, disminuida por los cambios hormonales existentes ${ }^{2}$.

Los índices de caries dental en embarazadas varían en Latinoamérica dependiendo del país, en Brasil la caries dental en embarazadas llega a $84,9 \%$ con un índice CPOD de 4, $0^{\circ}$; en Colombia se reporta una prevalencia del 82,8\% con índice CPOD del 6,69 ${ }^{10}$, en un reporte del 2011 en Cuba se relata una prevalencia del 80,95\% de caries en gestantes ${ }^{11}$, que para el 2015 en el mismo país alcanzó el $62,7 \%$ con un promedio de índice CPOD de $2,5^{12}$, mientras que en Chile estos valores alcanzan el 37,6\% con un índice CPOD de 4,79 ${ }^{13}$.

Otros trastornos estomatognáticos que afectan con una incidencia alta a mujeres en estado de gravidez son las enfermedades del periodonto. Se ha denominado el término "gingivitis del embarazo" ${ }^{1}$ caracterizada por inflamación gingival en un $75 \%$ de pacientes gestantes ${ }^{4}$, entre el tercer y octavo mes de embarazo, asociado a cambios hormonales sumados a una higiene oral deficiente ${ }^{4-6}$. La condición más severa de enfermedades del periodonto, la periodontitis, afecta en un $30 \%$ a las mujeres embarazadas ${ }^{5,6}$, siendo la periodontitis crónica la de mayor prevalencia, con un incremento proporcional a la edad ${ }^{9,11}$.

El granuloma piógeno, definido como una lesión hiperplásica que aparece como reacción a la presencia de placa y cambios hormonales, afecta a este grupo de riesgo, a partir del tercer mes de gestación, presentando una prevalencia de hasta el 5\%, y entre todas las lesiones gingivales de $35 \%^{1,13,14}$.

La ausencia de conocimientos de prevención, está asociado a la presencia de problemas de salud sistémicos y locales, cuyos desenlaces constituyen costos de infraestructura, personal médico y sobre todo alteraciones en el curso de la vida de quienes las padecen. Por ello, resulta imperativo la necesidad de sumar esfuerzos, entre las unidades básicas de atención de salud, especialistas médicos y odontólogos, incluyendo por supuesto la academia como elemento formador, para encaminar procesos que busquen el bienestar integral de la paciente embarazada.

Así la presente revisión de la literatura pretende buscar, revisar, sintetizar y actualizar los conocimientos acerca de las patologías orales en la mujer gestante.

\section{Revisión de la literatura actual}

Se planteó una revisión bibliográfica de los artículos que analizaron diversas alteraciones bucales en mujeres gestantes, para lo cual se empleó Mendeley Desktop, software de apoyo para la gestión documental facilitando la importación y exportación y el compartimiento de documentos en grupos privados y públicos, dando soporte al trabajo de investigación en la red social científica.

La búsqueda bibliográfica se realizó en las bases de datos Medline, SciELO y Google Scholar, usando las palabras clave: "mujeres embarazadas", "enfermedades bucodentales", "salud bucal" y sus homólogos en inglés, combinadas en las cadenas de búsqueda con los conectores booleanos AND y OR, empleando como filtro "free full text", en el período comprendido entre 2011 y 2018, incluyendo estudios de casos, ensayos clínicos, metanálisis, revisiones sistemáticas y revisiones de literatura que presenten información de las patologías durante el estado de gravidez.

En la búsqueda de la literatura gris sobre el tema, se incluyó todos aquellos documentos aportados por diferentes sociedades y asociaciones profesionales que brindan información acerca de las diferentes patologías orales en la mujer gestante y aquellas reformas, leyes o estatutos de la República del Ecuador que aporten con información para dicha revisión.

El principal criterio de exclusión fueron todos los estudios que mencionaron enfermedades bucales en mujeres embarazadas con enfermedades sistémicas preexistentes.

De los 72 artículos obtenidos se suprimió todos los documentos duplicados. Se analizó el título y resumen de los documentos teniendo en cuenta el criterio de exclusión. Por mutuo acuerdo de los autores se decidió la selección definitiva de 42 artículos de los cuales siete 
corresponden a leyes o reformas internas del Ecuador, posteriormente se procedió a la descarga del texto completo de los artículos seleccionados, la lectura completa de los artículos confirmó su inclusión (Figura 1).

La información analizada se estructuró en varios subapartados; en los que describe los factores de riesgo, cambios hormonales, la relación en detalle de cada patología oral vinculada a la mujer gestante y la salud bucal de la mujer embarazada en el Ecuador.

\section{Factores de riesgo}

Uno de los factores de riesgo de las mujeres en estado de gestación para adquirir enfermedades bucales es la higiene oral deficiente, la cual está desencadenada por náuseas y vómitos generalmente matinales, que en unión a una técnica incorrecta de cepillado predisponen a este grupo de riesgo a sufrir enfermedades gingivales y periodontales ${ }^{3}$.

A esto se suma la dieta y su importante papel en la salud oral de la mujer embaraza ${ }^{12,15}$, donde el consumo frecuente de alimentos ricos en azúcar, frecuentemente empleado para mitigar los malestares asociados al em- barazo ${ }^{3}$ contribuye a la presencia de un alto índice de placa bacteriana en gestantes ${ }^{1}$ haciendo importante la ejecución de prácticas adecuadas de higiene bucal ${ }^{12,16}$.

La falta de información acerca de los cuidados bucales durante el embarazo guarda estrecha relación con la caries dental y las periodontopatías ${ }^{4}$ independiente del nivel de educación de la gestante ${ }^{1}$; evidenciado sin embargo, que un aumento en la escolaridad y nivel socioeconómico desencadena mayor acceso a la atención prenatal y facilita adoptar medidas de salud preventiva y curativa a nivel oral ${ }^{17}$ cuando el asesoramiento existe ${ }^{18}$.

\section{Cambios hormonales en el embarazo}

La relaxina, hormona que se excreta en la gestación, cuya función es relajar las articulaciones y facilitar la labor de parto ${ }^{19,20}$, actúa sobre el ligamento periodontal provocando una ligera movilidad dentaria facilitando la entrada de restos de comida y el depósito de placa dentobacteriana a este nivel, desencadenando la inflamación de tejidos blandos; cabe recalcar que esta movilidad no provoca pérdida de inserción, ni precisa tratamiento y remite en el posparto ${ }^{8}$.

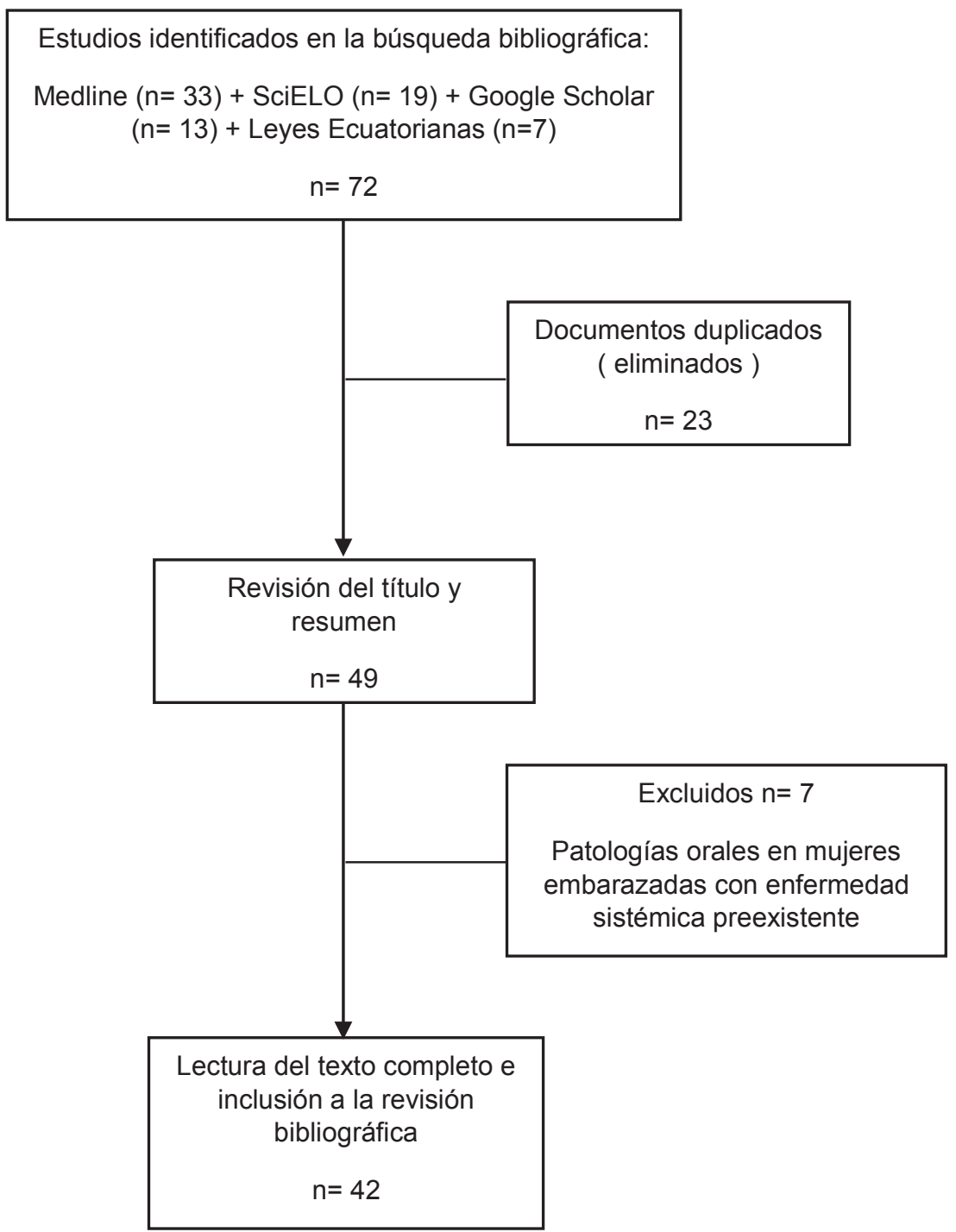

Figura 1. Proceso de selección de artículos en la revisión bibliográfica 
Aumento en los niveles hormonales, en especial de estrógenos y progesterona, esenciales para el desarrollo y desenvolvimiento del feto ${ }^{3}$ producen dilatación de los capilares y trae consigo la gingivitis del embarazo, que se caracteriza por edema, eritema, aumento del exudado gingival y de la permeabilidad capilar ${ }^{20-23}$.

La progesterona es la principal hormona del embarazo, encontrándose en niveles elevados durante todo el periodo gestacional ${ }^{24}$. Sus funciones más importantes son la preparación del endometrio para la implantación, además de mantener al mínimo la respuesta inmunológica del huésped para evitar afectación al feto ${ }^{23}$. Sin embargo, muestra de igual manera efectos adversos múltiples en la gestante tales como el aumento de riesgo de enfermedades cardiovasculares, migrañas, depresión e inclusive odontológicamente se la relaciona con gingivitis ${ }^{24}$. Esto se debe a diversos mecanismos, tales como un incremento de microorganismos anaerobios, aumento de interleuquinas inflamatorias, afectación de la fagocitosis de leucocitos y estimulación proteolítica enzimática ${ }^{23-26}$.

Los estrógenos son hormonas fundamentales que, en el ciclo gestacional, se mantienen en un nivel elevado, siendo producidas considerablemente a partir de la octava semana de embarazo ${ }^{23,26}$. Sus funciones, además de la producción de caracteres sexuales, son la estimulación de crecimiento uterino y el mantenimiento del flujo sanguíneo hacia la placenta. Los estrógenos, además producen efectos similares a los de la progesterona a nivel gingival, con aumento de la permeabilidad vascular y por consiguiente el edema ${ }^{25,26}$.

Los cambios considerables dentro del sistema inmunológico son evidentes, los estrógenos disminuyen la queratinización del epitelio gingival que conduce a la disminución de la barrera epitelial y por tanto a una mayor respuesta inflamatoria a los irritantes de la placa 1,2,24 contribuyendo al desarrollo de gingivitis grávida. La disminución quimiotáxica, reducción de linfocitos T mediadores de inflamación, estimulación fibroblástica y afección a peroxidasas, predisponen a la presencia de gingivitis, junto con una deficiente higiene oral, pudiendo desencadenar inclusive en una periodontitis ${ }^{15,23,26}$.

\section{Caries y embarazo}

Pese a la multifactorialidad de la caries dental, la disminución del $\mathrm{pH}$ en la saliva es considerada responsable de la presencia de caries en mujeres embarazadas ${ }^{9}$. Este aumento de acidez proporcional al avance del embarazo ${ }^{25}$ complementado con frecuente falta de higiene en la gestante como resultado de la disminución de la frecuencia del cepillado asociado al malestar general ${ }^{24}$ y a las náuseas favorece el desarrollo de bacterias, permitiendo el aumento en cantidad y extensión de placa dentobacteriana ${ }^{1,3}$.

\section{Enfermedad periodontal y embarazo}

La enfermedad periodontal ha sido considerada como una manifestación inflamatoria que afecta las estructuras blandas y duras, sostén de los dientes ${ }^{2}$. La gingi- vitis, etapa temprana de la enfermedad periodontal, se manifiesta con encías inflamadas y eritematosas ${ }^{16}$, la periodontitis, por otro lado, constituye la forma más grave de enfermedad periodontal ${ }^{22}$ caracterizada por la destrucción de las encías ${ }^{20}$, presente de forma más frecuente durante el segundo trimestre de embarazo ${ }^{2,23}$ y relacionada con el parto prematuro, nińos con bajo peso al nacer y preeclampsia ${ }^{25}$.

La enfermedad periodontal y la caries dental constituyen las lesiones más frecuentes a nivel bucal ${ }^{3,7,23}$; su presencia sin embargo, no es especifica de la mujer en esta etapa ${ }^{8}$ por lo que medidas de salud pública requieren establecerse y reforzarse de forma habitual, independiente de la edad y estado de gestación o no, considerando el riesgo ${ }^{5}$ y las implicaciones funcionales, sistémicas ${ }^{20} \mathrm{y}$ sicosociales de su presencia ${ }^{27,28}$, ya que las lesiones preexistentes pueden llegar a agudizarse comprometiendo la salud, la vida y calidad de vida de la madre y del feto ${ }^{29,30}$.

\section{Granuloma piógeno}

El granuloma piógeno también conocido como "hemangioma eruptivo", "hemangioma de granulación del tejido tisular", "granuloma gravídico", "hemangioma capilar lobular" y "tumor del embarazo" 29,30 constituye una lesión oral asociada a un sobrecrecimiento de tejido debido a irritación, trauma físico o factores hormonales 10,31. La gran vascularización presente en las lesiones recientes hacen que clínicamente se presente de color rojo, que cambia a rosa violeta en aquellas más antiguas, lisa o lobulada ${ }^{32}$, puede presentarse como una masa pediculada o sésil con base amplia ${ }^{17-19}$. Visible principalmente en la encía, pero apreciable también en otros sitios de la cavidad oral, como en el labio inferior, lengua, mucosa bucal, labio superior o paladar ${ }^{21}$, con una prevalencia del $1 \%$ en mujeres embarazadas ${ }^{33}$ con un origen asociado a la combinación de factores hormonales ${ }^{26,34}$, patógenos periodontales e irritantes locales, muy típicos en la cavidad bucal de una mujer embarazada ${ }^{15,27,33}$.

\section{Riesgos perinatales y salud bucal}

El nacimiento prematuro (PTB) es de relevancia mundial ya que aumenta las tasas de morbilidad y mortalidad neonatal ${ }^{30,31}$ de forma más frecuente en países menos desarrollados ${ }^{29}$. Su presencia está relacionada con infecciones maternas, incluyendo las de origen oral, desencadenantes de respuestas inflamatorias en la madre o el feto y una cascada de eventos que resultarían en PTB ${ }^{33}$ e hipoplasia del esmalte en los dientes temporales ${ }^{6,7,31}$.

\section{Acceso a la atención odontológica de la mujer embarazada}

El desconocimiento y falta de información oportuna constituye la causa de un bajo acceso de la mujer gestante a servicios de salud oral ${ }^{11,18}$, haciéndose necesario una verdadera concientización a las gestantes por parte de los profesionales de la salud ${ }^{9,22}$ sobre las diversas patologías que se pueden presentar, la práctica de técnicas de limpieza oral, la importancia de la atención a tiempo y las repercusiones en la salud de sus hijos ${ }^{27}$. 
Alrededor del 50\% de mujeres embarazadas no acuden a visita dental durante el embarazo, incluso cuando perciben algún malestar dental o bucal ${ }^{29}$.

\section{Las políticas de atención bucal de las gestantes en Ecuador}

En el Ecuador, las enfermedades bucodentales se encuentran entre las enfermedades de mayor demanda de atención en los servicios médicos del país, donde la caries dental es una de las patologías más prevalentes, siendo considerada como un problema de salud pública ${ }^{6}$. Las maternidades y clínicas del país reportan nacimiento de alrededor de 229476 niños y nińas nacidos vivos por mes ${ }^{30}$, lo que nos lleva a pensar que la población gestante alcanza un número elevado y representativo que potencialmente se convierte en recurrente de los servicios de salud pública y específicamente de los servicios de odontología ${ }^{35}$.

Las leyes ecuatorianas disponen que la salud es un derecho garantizado por el estado, mediante políticas económicas, sociales y servicios de promoción y atención integral de salud mediante la prestación de servicios regidos por principios de equidad, universalidad, solidaridad, interculturalidad, calidad, eficiencia, eficacia, precaución y bioética ${ }^{36-38}$. En este sentido, el modelo de Atención Integral de Salud (MAIS) propone un fortalecimiento del primer nivel de atención como puerta de entrada al sistema, basado en atención integral con continuidad y con un enfoque familiar, comunitario e individual ${ }^{39}$.

La creencia de que la mujer embarazada no puede recibir atención estomatológica aún es frecuentey es desencadenante de efectos adversos sobre los tejidos de la cavidad oral ${ }^{9}$ donde el personal médico, específicamente el ginecólogo, cumple un rol determinante ${ }^{19}$ por lo que un trabajo en conjunto con el odontólogo requiere ser planficado ${ }^{20}$.

Se constituye un desafío para los profesionales de salud contribuir a establecer prácticas de vida saludable para la mujer antes de la concepción y durante esta, y desde el inicio de la vida del futuro ser, relacionadas a una alimentación saludable, el consumo de agua potable y la actividad física ${ }^{40-43}$.

\section{Conclusiones}

La mujer embarazada sufre una serie de cambios durante el período de gestación, entre estos cambios están los hormonales, que pueden dar paso al progreso de diferentes patologías orales. Se requiere como profesionales de la salud conocer las implicaciones y difundirlas entre la población.

\section{Agradecimientos}

Agradecemos a la Universidad Tecnológica Equinoccial por los aportes y la facilidad que han brindado para realizar el presente trabajo.

\section{Referencias bibliográficas}

1. Corbella F, Taschieri S, Del Fabbro M, Francetti L, Weinstein R, Ferrazzi E. Adverse pregnancy outcomes and periodontitis: A systemic review and meta-analysis exploring potential association. Quintessence Int. 2016 Mar;47(3):193-204.

2. Gupta R, Acharya AK. Oral Health Status and Treatment Needs among Pregnant Women of Raichur District, India: A Population Based Cross-Sectional Study. Scientifica [Internet]. 2016 [Citado el 5 de enero del 2018] ID. 9860387, 8 páginas, 2016. DOI:10.1155/2016/9860387. Disponible en: https:// www.hindawi.com/journals/scientifica/2016/9860387/

3. Papanou PN. Systemic effects of periodontitis: lessons learned from research on atherosclerotic vascular disease and adverse pregnancy outcomes. Int Dent J. 2015 Dec; 65(6): 283-291. DOI: 0.1111/idj.12185

4. Yang M, Larcher P, Bueno-Silva B, Pinto M. Mechanisms involved in the association between periodontitis and complications in pregnancy. Front. Public health [Internet]. 2015 Jan [Citado el 7 de enero del 2018]. DOI: 10.3389/fpubh.2014.00290. Disponible en: https://www.frontiersin.org/articles/10.3389/fpubh.2014.00290/full

5. Vanterpool S, Tomsin K, Reyes L, Zimmermann L, Kramer B, Been J. Risk of adverse pregnancy outcomes in women with periodontal disease and the effectiveness of interventions in decreasing this risk: protocol for systematic overview of systematic reviews. Systematic Reviews [Internet]. 2016; 5(16) [Citado 7 de enero del 2018] DOI: 10.1186/s13643-016-0195-7.Disponible en: https://systematicreviewsjournal.biomedcentral. com/articles/10.1186/s13643-016-0195-7

6. Parihar AS, Katoch V, Rajguru SA, Rajpoot N, Singh P, Wakhle S. Periodontal disease: A possible risk-factor for adverse pregnancy outcome. J Int Oral Health. 2015 Jul;7(7):137-142

7. Penova-Veselinovic B, Keelan JA, Wang CA, Newnham JP, Penell CE. Changes in inflammatory mediators in gingival crevicular fluid following periodontal disease treatment in pregnancy: relationship to adverse pregnancy outcome. J Reprod Immunol. 2015 Nov; 112:1-10. DOI: $10.1016 /$ j.jri.2015.05.002

8. Schwendicke F, Karimbux N, Allareddy V, Gluud C. Periodontal treatment for preventing adverse pregnancy outcomes: A meta- and trial sequential analysis. PLoS ONE [Internet]. 2015 Jun. [Citado el 10 de enero del 2018] 10 (6): e0129060. DOI: 10.1371/journal. pone.0129060. Disponible en: http://journals.plos.org/ plosone/article?id=10.1371/journal.pone. 0129060

9. De Aguiar TC, Junior AV, Correa SR, Lopez F, Da Silva EP. Evaluación de factores de riesgo de padecer caries dentales en gestantes en Araquara, Brasil. Rev Cubana Estomatol. 2011;48(4):341-351.

10. Corchuelo J, Soto L, Villavicencio J. Situación de caries, gingivitis e higiene oral en gestantes y no gestantes en trece hospitales del Valle del Cauca. 2017;19(1):67-74. DOI: http://dx.doi.org/10.22267/rus.171901.70 
11. Gómez Porcegué Yillian, Macías Estrada Leonel. Salud Bucal en un grupo de Embarazadas. Área Sur. Sancti Spíritus. 2014. Gac Méd Espirit. 2015;17(3):48-55.

12. Perez AC, Valladares MB, Napolés NE, Naranjo MM, González B. Caries dental asociada a factores de riesgo durante el embarazo. Rev Cubana Estomatol. 2011;48(2):104-112.

13. Corisini G, Zaror C, Vallejos C. Uso de modelos epidemiológicos para estimar la incidencia de caries dental y enfermedad periodontal en embarazadas chilenas. Rev Clin Periodoncia Implantol Rehabil Oral. 2012;5(2):7477. DOI: $10.4067 /$ S0719-01072012000200004.

14. Adusumilli S, Yalamanchili PS, Manthena S. Pyogenic granuloma near the midline of the oral cavity: A series of case reports.J Indian Soc Periodontol. 2014;18(2):236239. DOI: 10.4103/0972-124X.131339

15. Marchesan JT, Morelli T, Moss K, Preisser JS, Zandona AF, Offenbacher S, Beck J. Interdental Cleaning Is Associated with Decreased Oral Disease Prevalence. J Dent Res [Internet]. 2018 Feb. [Citado 12 de febrero del 2018] DOI: 10.1177/0022034518759915. Disponible en: http://journals.sagepub.com/doi/ pdf/10.1177/0022034518759915

16. Johnson M, George A, Dahlen H, Ajwani S, Bhole $S$, Blinkhorn A, et al. The midwifery initiated oral health-dental service protocol: an intervention to improve oral health outcomes for pregnant women. BMC Oral Health. [Internet] 2015 [Citado el 22 de enero del 2018];15:2. DOI: 10.1186/1472-6831-15-2. Disponible en: https://bmcoralhealth.biomedcentral.com/ articles/10.1186/1472-6831-15-2

17. Zhong C, Ma KN, Wong YS, So Y, Lee PC, Yang Y. Oral Health Knowledge of Pregnant Women on Pregnancy Gingivitis and Children's Oral Health. J Clin Pediatr Dent. 2015;39(2):105-8. DOI: 10.17796/ jcpd.39.2.n66w635638w643n7

18. Miyoshi J, Ohba T, Ohkuma M, Katoh T, Tanoue D, Katabuchi H. Efficacy of a prospective community-based intervention to prevent preterm birth. J Perinat Med. 2017 Jan;45(1):113-119. DOI: 10.1515/jpm-2015-0408

19. Wagner Y, Heinrich-Weltzien R. Midwives' oral health recommendations for pregnant women, infants and young children: results of a nationwide survey in Germany. BMC Oral Health [Internet]. 2016 [Citado el 22 de enero del 2018]; 16:36. DOI: 10.1186/s12903-0160192-1. Disponible en: https://bmcoralhealth.biomedcentral.com/articles/10.1186/s12903-016-0192-1

20. Riggs E, Yelland J, Shankumar R, Kilpatrick N. We are all scared for the baby: promoting access to dental services for refugee background women during pregnancy. BMC Pregnancy Childbirth [Internet]. 2016 [Citado el 22 de enero del 2018];16:12. DOI: 10.1186/s12884015-0787-6. Disponible en: https://bmcpregnancychildbirth.biomedcentral.com/articles/10.1186/s12884015-0787-6

21. Hartnett E, Haber J, Krainovich-Miller B, Bella A, Vasilyeva A, Kessler J. Oral Health in Pregnancy. JOGNN. 2016;(5):565-573. DOI: 10.1016/j.jogn.2016.04.005

22. Marchesan JT, Morelli T, Moss K, Preisser JS, Zandona $\mathrm{AF}$, Offenbacher $\mathrm{S}$, Beck J. Interdental Cleaning Is
Associated with Decreased Oral Disease Prevalence. J Dent Res [Internet]. 2018 Feb. [Citado 12 de febrero del 2018] DOI: 10.1177/0022034518759915. Disponible en: http://journals.sagepub.com/doi/ pdf/10.1177/0022034518759915

23. Soares DM, de Moraes Ramos-Perez FM, de Araújo SS, Correia Leite de Marcelos PG, Dos Anjos Pontual A, da Cruz Perez DE. Sildenafil citrate on experimental periodontitis in rats: Microtomographic and histological analyses. Oral Dis. [Internet] 2018 Feb 26. [Citado el 01 de marzo del 2018] DOI: 10.1111/odi.12846. Disponible en: http://onlinelibrary.wiley.com/doi/10.1111/ odi.12846/abstract

24. Soucy-Giguére L, Tétu A, Gauthier S, Morand M, Chandad F, Gigére Y, et al. Periodontal disease and adverse pregnancy outcomes: A prospective study in a low risk population. J Obstet Gynaecol Can. 2016 Apr; 38(4):346-350. DOI: 10.1016/j.jogc.2016.02.012

25. Stelmakh V, Slot DE, van der Weijden GA. Self-reported periodontal conditions among dutch women during pregnancy. Int J Dent Hyg. 2017 Nov;15(4):e9-e15. DOI: $10.1111 /$ idh. 12210

26. Cohen L, Schaeffer M, Davideau JL, Tenenbaum H, Huck O. Obstetric knowledge, attitude, and behavior concerning periodontal diseases and treatment needs in pregnancy: Influencing factors in France. J Periodontol. 2015 Mar; 86(3):398-405. DOI: 10.1902/ jop.2014.140371

27. Gümüş P, Emingil G, Öztürk V-Ö, Belibasakis GN, Bostanci N. Oxidative stress markers in saliva and periodontal disease status: modulation during pregnancy and postpartum. BMC Infect Dis [Internet]. 2015 [Citado el 22 de enero del 2018]; 15:261. DOI: 10.1186/s12879015-1003-z. Disponible en: https://bmcinfectdis.biomedcentral.com/articles/10.1186/s12879-015-1003-z

28. Jain K, Kaur H. Prevalence of oral lesions and measurement of salivary $\mathrm{pH}$ in the different trimesters of pregnancy. Singapore Med J. 2015;56(1):53-57. DOI: 10.11 períndice622/smedj. 2015010

29. Díaz L, Valle R. Influencia de la salud bucal durante el embarazo en la salud del futuro bebé. Gac Méd Espirit [Internet]. 2015 Abr [citado 2018 Mayo 28] ; 17(1): 111-125.

30. Saxena P, Gupta S, Yadav S, Jain S, Jain D, Kamthan $S$, Saxena P. Oral health of pregnant females in central India: Knowledge, awareness, and present status. J Edu Health Promot. 2017;6:102. DOI: 10.4103/jehp. jehp_146_16.

31. Rocha J, Arima L, Werneck R, Moysés S, Baldani M. Determinants of Dental Care Attendance during Pregnancy: A Systematic Review. Caries Res. 2018;52:139152. DOI: $10.1159 / 000481407$.

32. Reddy NR, Kumar PM, Selvi T, Nalini HE. Management of Recurrent Post-partum Pregnancy Tumor with Localized Chronic Periodontitis. Int J Prev Med. 2014;5(5):643-647

33. Thomas E, Alves C, Ribeiro C, Batista R, Simóes V, Cavalli $\mathrm{R}$ et al. Perinatal outcomes and changes in the oral cavity: Brazilian cohorts of Ribeirão Preto and São Luís. Rev Bras Epidemiol. 2015;18(4):966-970. DOI: $10.1590 / 1980-5497201500040023$ 
34. Guastella C, Rinaldi V, Di Pasquale D, Coviello DA, Pignataro L. Oral pyoolitagenic granuloma gravidarum: A case report describing a large bleeding linguompletar informeal lesion. J Obstet Gynaecol. 2017 May; 37(4):537538. DOI: $10.1080 / 01443615.2016 .1256957$

35. Instituto Nacional de Estadística y Censos. Anuario de nacimientos y defunciones 2014. INEC; 2014 Disponible en: http://www.ecuadorencifras.gob.ec// documentos/web-inec/Poblacion_y_Demografia/Nacimientos_Defunciones/Publicaciones/Anuario_Nacimientos_y_Defunciones_2014.pdf

36. Steinberg B, Hilton I, Lada, Samelson R. Oral Health and Dental Care During Pregnancy. Dental theclinics. 57 (2013) 195-210. DOI: http://dx.doi.org/10.1016/j. cden.2013.01.002

37. Jiang H, Xiong X, Buekens P, Su Y, Qian X. Use of mouth rinse during pregnancy to improve birth and neonatal outcomes: a randomized controlled trial.BMC Pregnancy Childbirth. [Internet] 2015 [Citado 22 de enero del 2018]; 15:311. DOI: 10.1186/s12884-015-0761-3. Disponible en: https://bmcpregnancychildbirth.biomedcentral.com/articles/10.1186/s12884-015-0761-3

38. Asamblea constituyente del Ecuador. Constitución de la República del Ecuador. Quito; 2008. Disponible en: https://www.oas.org/juridico/pdfs/mesicic4_ecu_const.pdf
39. Asamblea constituyente del Ecuador. Ley orgánica de salud y su Reglamento -Decreto 1395. Quito; 2008. Disponible en: http://extwprlegs1.fao.org/docs/pdf/ ecu154958.pdf

40. Ministerio de Salud Pública del Ecuador. Modelo de Atención Integral del Sistema Nacional de Salud (MAIS). Quito; 2012. Disponible en: http://instituciones.msp.gob.ec/somossalud/images/documentos/guia/ Manual_MAIS-MSP12.12.12.pdf.

41. Ministerio de Salud Publica del Ecuador. Alimentacion y nutrición de la mujer gestante y de la madre en periodo de lactancia, Guia de Practica Clínica (GPC) Quito;2014. Disponible en: http://instituciones.msp.gob.ec/documentos/Guias/guias\%202014/Alimentacion\%20y\%20nutricion $\% 20 \mathrm{de} \% 201 \mathrm{a} \% 20$ madre\%2025-11-14.pdf

42. Ministerio de Inclusión Económica y Social. Desarrollo infantil para el buen vivir, un análisis para la política pública. Quito; 2016. Disponible en: http://www.inclusion.gob.ec/wp-content/uploads/downloads/2013/11/ Libro-de-Pol\%C3\%ADticas-P\%C3\%BAblicas.pdf

43. Ministerio de Salud Pública del Ecuador/Instituto Nacional de Estadística y Censos. Encuesta nacional de salud y nutrición de la población ecuatoriana de cero a 59 años. Ensanut-Ecu. Quito; 2014. Disponible en: https://www. unicef.org/ecuador/ENSANUT_2011-2013_tomo_1.pdf 
